\title{
Erratum to: Surface layer proteins from virulent Clostridium difficile ribotypes exhibit signatures of positive selection with consequences for innate immune response
}

Mark Lynch ${ }^{1,2}$, Thomas A. Walsh², Izabela Marszalowska ${ }^{1}$, Andrew E. Webb², Micheál Mac Aogáin ${ }^{3}$, Thomas R. Rogers ${ }^{3}$, Henry Windle ${ }^{4}$, Dermot Kelleher ${ }^{5}$, Mary J. O'Connell ${ }^{2,6^{* \dagger}}$ and Christine E. Loscher ${ }^{1 \dagger}$

\section{Erratum}

"Upon publication of the original article [1], it was noticed that there was an error in the author name. The author's name should be "Micheál Mac Aogáin" instead of Micheál MacAogain."

\begin{abstract}
Author details
${ }^{1}$ Immunomodulation Research Group, School of Biotechnology, Dublin City University, Glasnevin, Dublin 9, Ireland. ${ }^{2}$ Bioinformatics and Molecular Evolution Group, School of Biotechnology, Dublin City University, Glasnevin, Dublin 9, Ireland. ${ }^{3}$ Department of Clinical Microbiology, Trinity College Dublin, St James Hospital Dublin, Dublin, Ireland. ${ }^{4}$ Institute of Molecular Medicine, Trinity College Dublin, Dublin, Ireland. ${ }^{5}$ Faculty of Medicine, Imperial College London, London SW7 2AZ, UK. ${ }^{6}$ Computational and Molecular Evolutionary Biology Research Group, School of Biology, Faculty of Biological Sciences, The University of Leeds, LeedsLS2 9JTUK.
\end{abstract}

Received: 1 May 2017 Accepted: 1 June 2017

Published online: 12 June 2017

\section{Reference}

1. Surface layer proteins from virulent Clostridium difficile ribotypes exhibit signatures of positive selection with consequences for innate immune response. BMC Evolutionary Biology 2017 17:90. DOI 10.1186/s12862-0170937-8

\footnotetext{
* Correspondence: m.oconnell@leeds.ac.uk

${ }^{\dagger}$ Equal contributors

${ }^{2}$ Bioinformatics and Molecular Evolution Group, School of Biotechnology,

Dublin City University, Glasnevin, Dublin 9, Ireland

${ }^{6}$ Computational and Molecular Evolutionary Biology Research Group, School

of Biology, Faculty of Biological Sciences, The University of Leeds, LeedsLS2

9JTUK

Full list of author information is available at the end of the article
} 